\title{
Delayed removal of a maxillary third molar from the infratemporal fossa
}

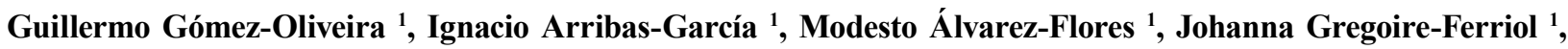 \\ Carlos Martínez-Gimeno ${ }^{2}$
}

${ }^{1} \mathrm{MD}$, Consultant of Oral and Maxillofacial Surgery Department. Hospital Universitario de Canarias

${ }^{2}$ Head of Oral and Maxillofacial Surgery Department. Hospital Universitario de Canarias

Correspondence:

Hospital Universitario de Canarias

Servicio de Cirugía Oral y Maxilofacial

Ofra s/n. La Cuesta

38320 La Laguna (Tenerife) Spain

ggomoli@hotmail.com

Gómez-Oliveira G, Arribas-García I, Álvarez-Flores M, Gregoire-Ferriol J, Martínez-Gimeno C. Delayed removal of a maxillary third molar from the infratemporal fossa. Med Oral Patol Oral Cir Bucal. 2010 May 1;15

\section{(3):e509-11.}

http://www.medicinaoral.com/medoralfree01/v15i3/medoralv15i3p509.pdf

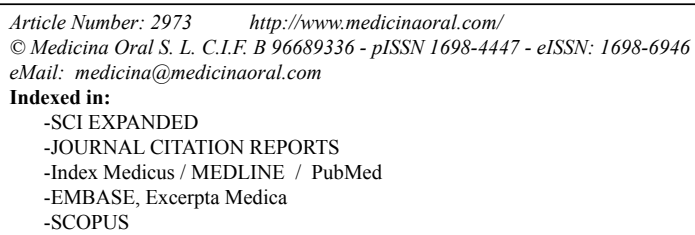

\begin{abstract}
Removal of an impacted superior third molar is a simple and easy procedure for an Oral and Maxillofacial Surgeon. Nevertheless, complications are possible and include infection, facial swallowing, trismus, wound dehiscence, root fracture or even orosinusal fistula. Iatrogenic displacement into the infratemporal fossa is frequently mentioned but rarely reported.

This anatomical fossa includes several important structures such as the internal maxillary artery, the venous pterygoid plexus, the sphenopallatinun nerve, the coronoid process of the mandible and the pterygoid muscles. Recommended treatment includes immediate surgical removal if possible or initial observation and secondary removal, as necessary, because of infection, limited mandibular motion, inability to extract the tooth, or the patient's psychologic unease. Sometimes, the displaced tooth may spontaneously migrate inferiorly and becomes accessible intraorally.

This report describes the location and secondary surgical removal of a left maxillary third molar displaced into the infratemporal fossa, two weeks after first attempt of extraction.
\end{abstract}

Key words: Third molar, complication, displacement, infratemporal fossa. 


\section{Introduction}

The surgical removal of impacted maxillary third molars is a procedure routinely carried out by Oral and Maxillofacial Surgeons, and it is usually associated with low rates of complications and morbidity (1). These complications include fracture of the maxillary tuberosity, tooth root fracture, perforation of the maxillary sinus, prolapse of the buccal fat pad and displacement of the roots or tooth into the maxillary sinus (2).

Displacement of a maxillary third molar into the infratemporal fossa is frequently mentioned but rarely reported (1-3). Imaging and retrieval of such a displaced tooth is difficult (1). Some authors recommend urgent hospitalization and removal of the tooth under general anesthesia with the aid of image intensification (1) while others have suggested removal via a transantral or a coronal approach $(3,4)$.

Prevention of displacement with the use of a distal retractor is recommended when surgically removing of impacted maxillary third molars are performed (1).

\section{Case Report}

A healthy 23-year-old woman was referred to our consultation for removal of four impacted third molars. After explanation of the surgical procedure, the patient signed the informed consent and underwent surgical removal of the third molars under local anesthesia. Either both inferior and right superior third molars were removal uneventfully. The maxillary left third molar was then approached with a typical incision over the tuberosity that extended into the buccal gingival margin of the second molar until the crown of the tooth could be visualized. A straight elevator was then used to remove the tooth. During luxation, the tooth inadvertently slid distally into the infratemporal fossa.

Immediately, the incision was extended distally to allow exposure of the posterolateral aspect of the maxillary wall. After several attempts and, although it could be palpated lateral to the pterygoid plates and below the zygomatic arch, such tooth could not be removed. Then, a panoramic radiograph (Fig. 1) as well as a 3D CT -scan was performed and showed the third molar to be located into the infratemporal fossa, just between zygomatic arch and lateral pterygoid plate. (Fig. 2).

Alternatives of treatment, either surgical or conservative, were discussed with the patient who decided a "wait and see" option. Then, the previously incised mucosa was closed with a resorbable suture and the patient was placed on amoxicillin-clavulanic $875 \mathrm{mg}$ every 8 hours and an analgesic for two weeks and referred again to our consultation.

Two weeks later, the patient was asymptomatic, there was no limitation of the mouth opening and the tooth was palpated submucosal distally to the second molar. Accordingly with the patient, it was decided to remove

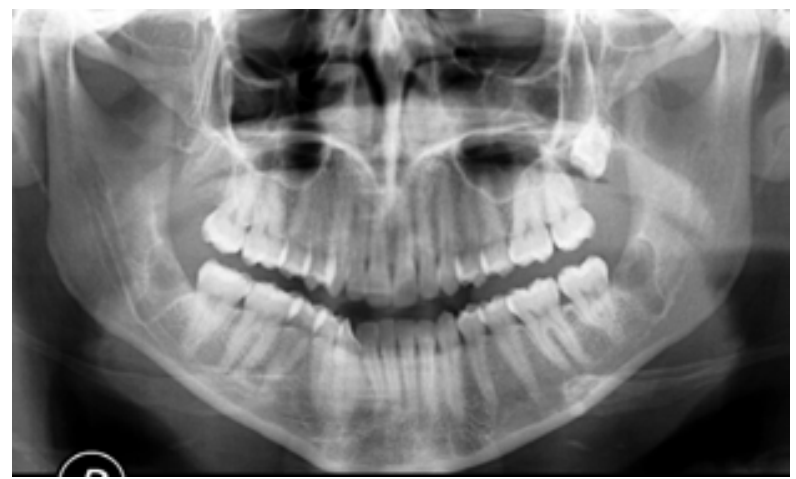

Fig. 1. Panoramic radiograph showing the third molar displaced. Note that this radiograph does not allow neither plan the surgery nor locating the exact position of the tooth.

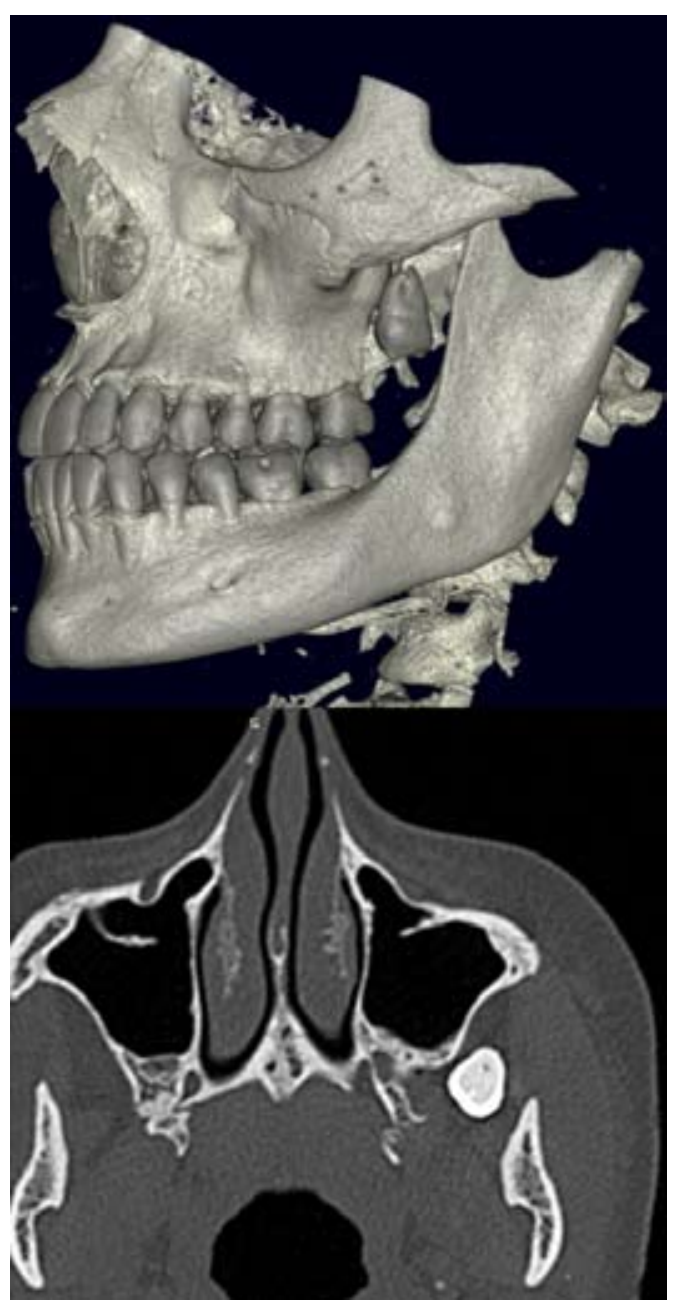

Fig. 2. CT-scan showing the maxillary third molar located into the infratemporal fossa and its relationship to the zygomatic arch and the pterygoid plates. 
the tooth via the previously intraoral incision under local anesthesia. The tooth was removed uneventfully and the patient was discharged to her house.

One month after surgery, the patient remained asymptomatic and was definitively discharged from the Consultation.

\section{Discussion}

Displacement of maxillary third molars into the infratemporal fossa is usually associated with an incorrect extraction technique, distolingual angulated tooth, decreased visibility during surgical removal or limited bone distal to the third molar (1).

The exact anatomic location of the displaced tooth is not easy to determine clinically $(2,5)$ what represents a surgical problem. These teeth usually displace through the periosteum into the infratemporal fossa just adjacent to the lateral pterygoid plate and inferior to the lateral pterygoid

muscle (5). Nevertheless, this position may change upwards into the skull base if the tooth is attempted to retrieve $(1,5)$.

Radiographic examination is indicated to locate the displaced tooth. Occlusal, panoramic, occipitomental or lateral radiographs can be used, although CT-scan is the powerful and useful technique, because it provides an exact and anatomical location $(2,5)$.

Clinically, the patient may be asymptomatic or has symptoms as swelling, pain, limitation of mandibular motion or even trismus, if fibrosis is present (2). In our case, the patient was always asymptomatic and with a normal range of mandibular motion.

Therapeutic attitude is based on clinical symptoms and signs, on surgeon preferences and, of course, on patient decision. The complex anatomy of the infratemporal fossa, the potential morbidity and the difficult to obtain a good surgical exposure of the region are limiting factors for the treatment (1). Complications may increase if the retrieval procedure is delayed such as infection, foreign body reaction or trismus (6).

According to some authors, displaced teeth can migrate downwards into the oral cavity, allowing an easy surgical removal $(1,6)$. Nevertheless, others, reported migration is impossible because of fibrosis and anatomic boundaries (4).

Several surgical approaches can be used such as coronal, Gillies, Caldwell-Luc or resection of the coronoid process (2-4). We preferred to postpone the retrieval surgery for two weeks according to some authors based on fibrous tissue formation is able to immobilize the tooth and on the possibility of an inferiorly displacement of the tooth. This delay also avoids the possible displacement of the tooth deeper to the skull base if an early retrieval attempt is performed (3). Elsewhere, we recommend performing a CT-scan in order to precisely locate the third molar and to plan the retrieval procedure.

One maneuver extremely recommended is to place a separator distal to the third molar while removing it, in order to avoid inadvertent migration to the infratemporal fossa.

\section{References}

1. Patel M, Down K. Accidental displacement of impacted maxillary third molars. Br Dent J. 1994;177:57-9.

2. Sverzut CE, Trivellato AE, Sverzut AT, de Matos FP, Kato RB.

Removal of a maxillary third molar accidentally displaced into the infratemporal fossa via intraoral approach under local anesthesia: report of a case. J Oral Maxillofac Surg. 2009;67:1316-20.

3. Orr DL 2nd. A technique for recovery of a third molar from the infratemporal fossa: case report. J Oral Maxillofac Surg. 1999;57:1459-61.

4. Gulbrandsen SR, Jackson IT, Turlington EG. Recovery of a maxillary third molar from the infratemporal space via a hemicoronal approach. J Oral Maxillofac Surg. 1987;45:279-82.

5. Oberman M, Horowitz I, Ramon Y. Accidental displacement of impacted maxillary third molars. Int J Oral Maxillofac Surg. 1986;15:756-8.

6. Dimitrakopoulos I, Papadaki M. Displacement of a maxillary third molar into the infratemporal fossa: case report. Quintessence Int. 2007;38:607-10. 